\title{
NOTE ON A THEOREM DUE TO BROMWICH
}

\author{
BY H. L. GARABEDIAN
}

The following well known theorem is due to Bromwich.*

Theorem. Suppose (i) that the series $\sum a_{n}$ is summable by Cesàro means of order $k$ to the sum $s$, (ii) that $v_{n}$ is a function of $x$ with the properties

$$
\left.\begin{array}{l}
\sum n^{k}\left|\Delta^{k+1} v_{n}\right|<K \dagger \\
\lim _{n \rightarrow \infty} n^{k} v_{n}=0
\end{array}\right\} \quad \text { if } \quad x>0,
$$

$$
\lim _{x \rightarrow 0} v_{n}=1 \text {, }
$$

where $K$ is independent of $x$ and $n$. Then the series $\sum a_{n} v_{n}$ converges if $x$ is positive, and

$$
\lim _{x \rightarrow 0} \sum a_{n} v_{n}=s .
$$

I propose to establish this theorem by a more direct and shorter method than that used by Bromwich. Moreover, this proof affords a method of exhibiting a $k$-fold summability with infinite matrix of reference, analogous to well known definitions of summability with finite matrices of reference which make use of repeated means, for any $v_{n}$ which satisfies the conditions of the theorem under discussion.

By hypothesis the series $\sum a_{n}$ is summable by Cesàro means of order $k$, so that if

$$
S_{n}^{(k)}=\left(\begin{array}{c}
n+k-1 \\
k-1
\end{array}\right) s_{0}+\left(\begin{array}{c}
n+k-2 \\
k-1
\end{array}\right) s_{1}+\cdots+\left(\begin{array}{c}
k-1 \\
k-1
\end{array}\right) s_{n}
$$

and

$$
A_{n}^{(k)}=\left(\begin{array}{c}
n+k \\
k
\end{array}\right)
$$

* Mathematische Annalen, vol. 65 (1907-08), pp. 350-369; p. 359.

$\dagger$ Since all of the terms in the series $\sum n^{k}\left|\Delta^{k+1} v_{n}\right|$ are positive, this condition implies the convergence of the series. 
then

$$
C_{n}^{(k)}=\frac{S_{n}^{(k)}}{A_{n}^{(k)}}
$$

has a definite limit $s$ as $n$ tends to $\infty$. We may also define $S_{n}^{(k)}$ by means of the identities

$$
\sum S_{n}^{(k)} x^{n}=(1-x)^{-k} \sum s_{n} x^{n}=(1-x)^{-(k+1)} \sum a_{n} x^{n},
$$

from which it follows that

$$
\sum s_{n} x^{n}=(1-x)^{k} \sum S_{n}^{(k)} x^{n}
$$

and

$$
\sum a_{n} x^{n}=(1-x)^{k+1} \sum S_{n}^{(k)} x^{n} .
$$

It results at once from the last identity that

$$
\begin{aligned}
a_{n}= & S_{n}^{(k)}-\left(\begin{array}{c}
k+1 \\
1
\end{array}\right) S_{n-1}^{(k)}+\left(\begin{array}{c}
k+1 \\
2
\end{array}\right) S_{n-2}^{(k)}-\cdots \\
& +(-1)^{k+1}\left(\begin{array}{c}
k+1 \\
k+1
\end{array}\right) S_{n-k-1}^{(k)}
\end{aligned}
$$

where it is understood that when a negative subscript occurs in the formula, the corresponding $S^{(k)}$ is to be replaced by zero.

Now, we form the series

$$
F(x)=\sum_{n=0}^{\infty} a_{n} v_{n}(x)
$$

or, using (1),

(2) $F(x)=\sum_{n=0}^{\infty}\left[S_{n}^{(k)}-\left(\begin{array}{c}k+1 \\ 1\end{array}\right) S_{n-1}^{(k)}+\left(\begin{array}{c}k+1 \\ 2\end{array}\right) S_{n-2}^{(k)}-\cdots\right.$

$$
\left.+(-1)^{k+1}\left(\begin{array}{l}
k+1 \\
k+1
\end{array}\right) S_{n-k-1}^{(k)}\right] v_{n}(x) \text {. }
$$

We are justified by the conditions (ii) in ordering the terms of (2) with respect to $S_{n}{ }^{(k)}$ to get 


$$
\begin{aligned}
F(x)= & \sum_{n=0}^{\infty} S_{n}^{(k)}\left[v_{n}(x)-\left(\begin{array}{c}
k+1 \\
1
\end{array}\right) v_{n+1}(x)+\cdots\right. \\
& \left.+(-1)^{r}\left(\begin{array}{c}
k+1 \\
r
\end{array}\right) v_{n+r}(x)+\cdots+(-1)^{k+1} v_{n+k+1}(x)\right]
\end{aligned}
$$

or

$$
F(x)=\sum_{n=0}^{\infty}\left(\begin{array}{c}
n+k \\
k
\end{array}\right) \Delta^{k+1} v_{n}(x) C_{n}^{(k)} .
$$

Since $\lim _{n \rightarrow \infty} C_{n}(k)=s$, it remains to show that the method of summation with infinite matrix of reference defined by (3) is regular, ${ }^{*}$ which is to say that $\lim _{x \rightarrow 0} F(x)=s$. Accordingly, we must require in the present case that

$$
\begin{aligned}
& \lim _{x \rightarrow 0}\left(\begin{array}{c}
n+k \\
k
\end{array}\right) \Delta^{k+1} v_{n}(x)=0 \text { for every } n, \\
& \lim _{x \rightarrow 0} \sum_{n=0}^{\infty}\left(\begin{array}{c}
n+k \\
k
\end{array}\right) \Delta^{k+1} v_{n}(x)=1, \\
& \sum_{n=0}^{\infty}\left(\begin{array}{c}
n+k \\
k
\end{array}\right)\left|\Delta^{k+1} v_{n}(x)\right|<K
\end{aligned}
$$

for every $x>0, K$ independent of $x$.

It follows from condition $(\alpha)$ of the hypotheses that

$$
\lim _{x \rightarrow 0}\left(\begin{array}{c}
n+k \\
k
\end{array}\right) \Delta^{k+1} v_{n}(x)=\left(\begin{array}{c}
n+k \\
k
\end{array}\right) \Delta^{k+1} 1=0 .
$$

Accordingly, the requirement (a) is satisfied.

Now, we need the identity

$$
\begin{gathered}
\sum_{n=0}^{n}\left(\begin{array}{c}
n+k \\
k
\end{array}\right) \Delta^{k+1} v_{n}(x)=v_{k}(x)+\sum_{\nu=0}^{k-1} \frac{k !}{(k-\nu) ! \nu !} \Delta^{k-\nu} v_{\nu}(x) \\
-\sum_{\nu=0}^{k} \frac{(n+k-\nu) !}{(k-\nu) ! n !} \Delta^{k-\nu} v_{n+1}(x) \cdot \dagger
\end{gathered}
$$

* See Carmichael, The theory of summable series, this Bulletin, vol. 25 (191819), pp. 97-131; p. 117.

† See H. L. Garabedian, Annals of Mathematics, vol. 32 (1930), pp. 83-106; p. 91. 
As $n$ tends to infinity every term involving $n$ on the right-hand side of (4) tends to zero by virtue of condition $(\beta)$ of the hypotheses. It follows that

(5) $\sum_{n=0}^{\infty}\left(\begin{array}{c}n+k \\ k\end{array}\right) \Delta^{k+1} v_{n}(x)=v_{k}(x)+\sum_{\nu=0}^{k-1} \frac{k !}{(k-\nu) ! \nu !} \Delta^{k-\nu} v_{\nu}(x)$.

Moreover, by virtue of condition $(\alpha)$,

$$
\lim _{x \rightarrow 0} \sum_{\nu=0}^{k-1} \frac{k !}{(k-\nu) ! \nu !} \Delta^{k-\nu} v_{\nu}(x)=0 .
$$

Accordingly, from (5), (6), and $(\alpha)$ we have

$$
\lim _{x \rightarrow 0} \sum_{n=0}^{\infty}\left(\begin{array}{c}
n+k \\
k
\end{array}\right) \Delta^{k+1} v_{n}(x)=\lim _{x \rightarrow 0} v_{k}(x)=1,
$$

and the requirement (b) is fulfilled.

Finally, we note that the expression

$$
\sum_{n=0}^{\infty}\left(\begin{array}{c}
n+k \\
k
\end{array}\right)\left|\Delta^{k+1} v_{n}(x)\right|
$$

will be uniformly bounded or fail to be uniformly bounded according as the expression $\sum_{n=0}^{\infty} n_{k}\left|\Delta^{k+1} v_{n}(x)\right|$ is uniformly bounded or fails to be uniformly bounded. It is understood in this statement that $x$ is restricted to positive values. Hence, by condition $(\gamma)$, the last of the requirements (c) for regularity is satisfied.

We conclude that

$$
\lim _{x \rightarrow 0} F(x)=\lim _{x \rightarrow 0} \sum_{n=0}^{\infty}\left(\begin{array}{c}
n+k \\
k
\end{array}\right) \Delta^{k+1} v_{n}(x) C_{n}^{(k)}=s .
$$

We exhibit in the function

$$
\phi(n, x)=\left(\begin{array}{c}
n+k \\
k
\end{array}\right) \Delta^{k+1} v_{n}(x)
$$

a convergence factor which, associated with a method of summation with infinite matrix of reference, affords a method of constructing a $k$-fold method of summability with infinite matrix of reference for any $v_{n}(x)$ which satisfies the conditions of Bromwich's theorem. Examples of functions $v_{n}(x)$ which satisfy these requirements* are the I.eRoy convergence factor:

* H. L. Garabedian, loc. cit. 


$$
v_{n}(x)=\frac{\Gamma[(1-x) n+1]}{\Gamma(1+n)}
$$

the Mittag-Leffler convergence factor:

$$
v_{n}(x)=\frac{1}{\Gamma(1+n x)} ;
$$

and the Dirichlet series convergence factors:

$$
v_{n}(x)=e^{-\lambda(n) x},
$$

where $\lambda(n)$ must be a logarithmico-exponential function of $n$ which tends to infinity with $n$ but not as slowly as $\log n$ nor faster than $n^{\Delta}$, where $\Delta$ is any constant however large.

NORTHWESTERN UNIVERSITY

\title{
A THEOREM ON SYMMETRIC DETERMINANTS
}

\author{
BY W. V. PARKER
}

1. Introduction. In a recent paper* the writer proved the following theorem.

If $D=\left|a_{i j}\right|$ is a real symmetric determinant of order $n, n>5$, in which $a_{i i}=0,(i=1,2, \cdots, n)$, and $M$ is any principal minor of $D$ of order $n-1$, then if all fourth order princifal minors of $M$ are zero, $D$ vanishes.

The purpose of the present note is to establish a second theorem of a similar nature which applies to complex as well as to real determinants. It will be shown also that when $a_{i j},(i \neq j)$, $(i, j=1,2, \cdots, n)$, is real and different from zero the conditions of this second theorem imply those of the above.

2. A Second Theorem. The theorem with which this note is concerned may be stated as follows.

TheOREM. If $D=\left|a_{i j}\right|$ is a symmetric determinant of order $n$, $n>5$, in which $a_{i i}=0,(i=1,2, \cdots, n)$, and $M$ is any principal minor of $D$ or order $n-1$, then if all fourth order principal minors of $D$, which are not minors of $M$, are zero, $D$ vanishes.

* This Bulletin, vol. 38 (1932), p. 259. 